\title{
Wake Acoustic Analysis and Image Decomposition via Beamforming of Microphone Signal Projections on Wavelet Subspaces
}

\author{
Hadi S. Wassaf* \\ John A. Volpe National Transportation Systems Center, Cambridge MA, 02142 \\ Yan Zhang ${ }^{\dagger}$ \\ Jet Propulsion Laboratory, Pasadena, CA, 91109 \\ and \\ Oliver C. Ibe \\ University of Massachusetts Lowell, Lowell, MA 01854
}

\begin{abstract}
This paper describes the integration of wavelet analysis and time-domain beamforming of microphone array output signals for analyzing the acoustic emissions from airplane generated wake vortices. This integrated process provides visual and quantitative simultaneous information about the wake signal composition and array resolution for a particular wavelet subspace during a time interval, $T$. In the results section, an example is given on how image processing algorithms might be used to automate the extraction of this information and select the wavelet subspaces from which to perform image reconstruction. This process begins with the projection of all the microphone signals on wavelet multiresolution subspaces. The projections of these signals on the same wavelet subspace or scale are then beamformed to produce an image of the wake corresponding to that particular scale. Therefore for each time interval $T$, the process produces a number of images equal to that of the wavelet scales. This is equivalent to a more conventional Fourier-based idea of filtering the microphone signals with band-pass filters having non-uniform bandwidths then beamform in different sub-bands, but offers greater flexibility and enhanced computational speed. Results from both approaches will be shown, which ultimately illustrate the advantages of wavelet analysis over that of the Fourier-based analysis. Amongst the advantages are the speed of the decomposition and ease of the image reconstruction from selected subspaces aided by the perfect reconstruction and orthogonality properties of wavelet analysis.
\end{abstract}

\section{Nomenclature}

$a_{j}(n) \quad=$ Coefficients of the projection of $\mathrm{f}(\mathrm{t})$ in $\mathrm{V}_{\mathrm{j}}$

$b_{j}(n) \quad=$ Coefficients of the projection of $\mathrm{f}(\mathrm{t})$ on $\mathrm{W}_{\mathrm{j}}$

$B \quad=$ Orthonormal basis for the decomposition comprised of $\mathrm{V}_{0}$ and $\mathrm{m}$ wavelet subspaces

c $\quad=$ Nominal speed of sound

$f_{j}(t) \quad=$ Approximation of a function $\mathrm{f}(\mathrm{t})$ in $\mathrm{V}_{\mathrm{j}}$

$F_{0}(n) \quad=$ Coefficients for the low-pass filter for the synthesis bank

$F_{l}(n) \quad=$ Coefficients for the high-pass filter for the synthesis bank

$h_{0}(k)=$ Coefficients of the refinement equation

$h_{l}(k)=$ Coefficients of the wavelet equation

$H_{0}(n)=$ Coefficients of the low-pass filter for the analysis bank

$\mathrm{H}_{1}(\mathrm{n}) \quad=$ Coefficients of the high-pass filter for the analysis bank

\footnotetext{
* Electronics Engineer, Advanced Communication Navigation and Surveillance Division, RTV-4A.

${ }^{\dagger}$ Senior Member Engineering Staff, Interferometry and Advanced Optical Systems Section.

\# Associate Professor, Department of Electrical and Computer Engineering, 


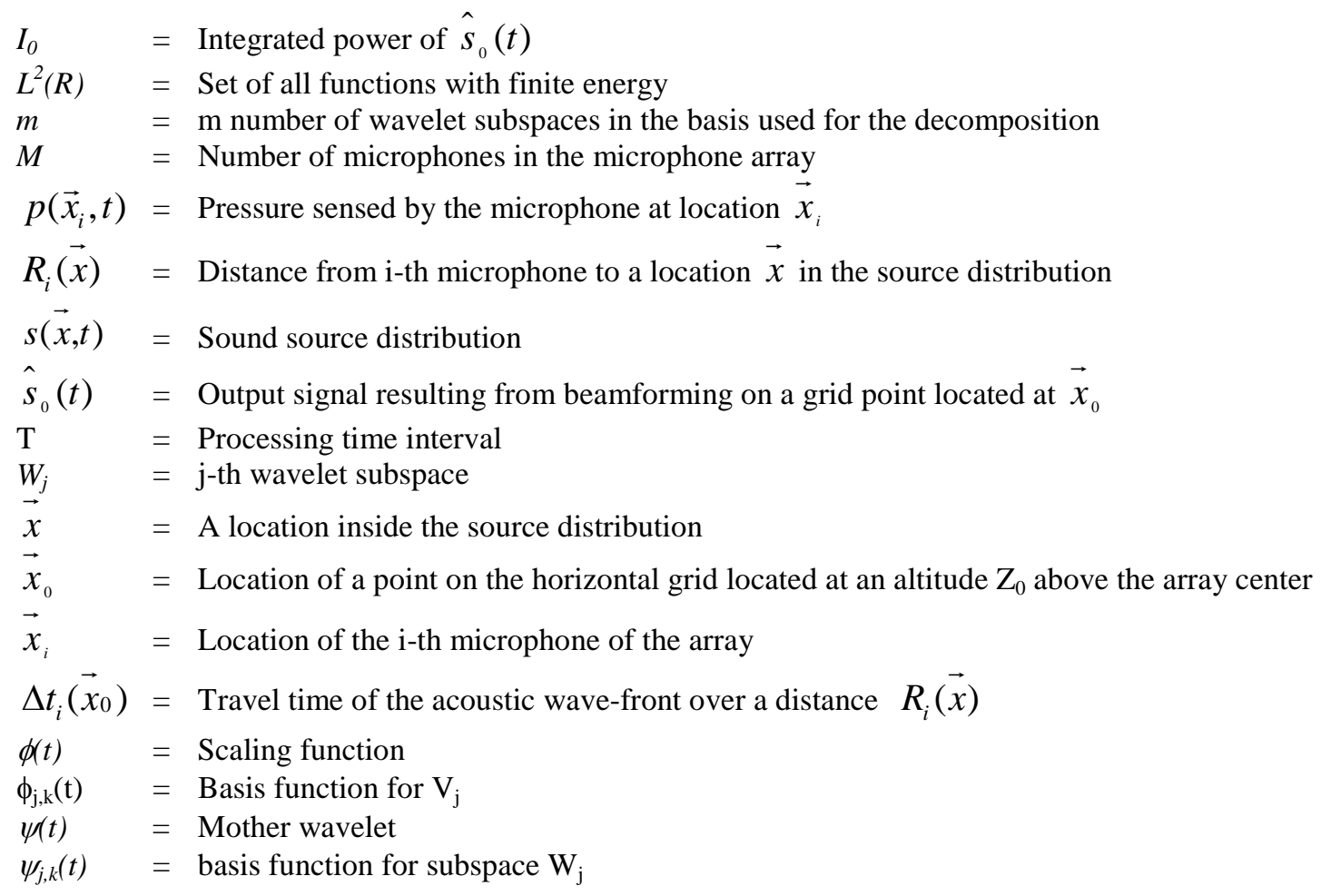

\section{Introduction}

The use of microphone arrays to visualize and track aircraft vortices represents a recent and novel development in both fundamental fluid dynamics and aeroacoustics as highlighted in a number of publications ${ }^{5,7}$. The state-of-theart wake acoustics knowledge has been advanced by using both time and frequency domain based algorithms to generate images representing Acoustic Intensity Distribution Maps (AIDM), otherwise also known in aeroacoustics as the source localization map. In these images the wakes appear as two bright lines behind the aircraft (as shown in Fig. 5 of the results section). More studies have also been carried out to enhance the imaging of the wakes and array resolution using deconvolution techniques ${ }^{8}$. These approaches have proved useful in enhancing vertical resolution and therefore 3-D imaging and tracking capabilities of microphone arrays. However, when information about the signal composition of the wake is desired, special processing of the raw signal and not of the images produced from broadband microphone signals is needed. One approach to getting this information is using frequency domain beamforming to produce AIDMs corresponding to multiple narrow analysis bands ${ }^{9}$. However, this approach becomes computationally expensive when the signal analyzed is broadband. This is because the steering vectors of the beamformer are a function of frequency and therefore the beamforming bands must be very narrow, which results in a large number of AIDMs to cover the whole spectrum to be analyzed. In this study a wavelet-based approach, coupled with both image processing and time domain beamforming, has been developed to analyze the composition of the wake signal, with the ability to reconstruct the image in any combination of wavelet subspaces (or sub-bands) without having to re-filter and re-beamform. This approach makes use of the multi-resolution, orthogonality and perfect reconstruction properties of the wavelet decomposition to generate classified beamforming images. The wavelet-based decomposition has advantages over Fourier-based filtering because it lends itself to an efficient filter bank implementation of the decomposition and reconstruction of the signal while guaranteeing orthogonality and perfect reconstruction (PR) of the original signal from its components. The orthogonality and PR properties of wavelet result in an efficient way of recomposing images from subspaces when needed, without having to re-beamform the raw signals.

The remainder of the paper is organized as follows. Section II-A provides a background on wavelets and shows the connection between wavelet analysis and filter banks. Section II-B discusses beamforming and how AIDMs are produced and concludes with a flow-chart illustrating the integration of beamforming and multiresolution wavelet 
analysis. Section III shows example applications of this approach and validation of results using a Fourier-based approach. Section IV describes future work and possible improvements to this technique.

\section{Background}

\section{A. Wavelet Multiresolution Analysis and Filter Banks}

This section introduces the concept of wavelets and how a signal could be decomposed into wavelet subspaces using a filter bank. A more detailed development of wavelet analysis could be found in References 1 and 3 . Let the process start by defining a set of nested spaces that are assumed complete in $L^{2}(R)$ when $m \rightarrow \infty\left(L^{2}(R)\right.$ is the set of all finite energy functions).

$$
\ldots \subset \mathrm{V}_{-\mathrm{n}} \subset \mathrm{V}_{-\mathrm{n}+1} \subset \mathrm{v}_{0} \subset \mathrm{V}_{1} \subset \mathrm{V}_{\mathrm{j}} \subset \mathrm{V}_{\mathrm{m}} \subset \ldots
$$

such that $\mathrm{V}_{\mathrm{j}}$ has a basis function $\phi_{j, k}(t)=\phi\left(2^{j} . t-k\right)$ where $\phi(\mathrm{t}) \in \mathrm{L}^{2}(\mathrm{R})$ satisfies the following equation called the refinement equation

$$
f(t)=2 \sum_{k=0}^{N} h_{0}(k) f(2 t-k)
$$

where

$$
\sum_{k=1}^{N}\left|h_{0}(k)\right|=1
$$

so that $\phi(\mathrm{t})$ is normalized to 1 . It can be shown that when this function exists it is compactly supported and is completely defined by the coefficients $h_{0}(k)$ and can be synthesized using an FIR filter whose coefficients are $h_{0}(k)$.

In this case any function $f(t)$ can be approximated by a projection on the space $\mathrm{V}_{\mathrm{j}}$ according to equation (3).

$$
f_{j}(t)=\sum_{k=0}^{\infty} a_{j}(k) \cdot \phi_{j, k}(t)
$$

where

$$
a_{j}(k)=\int_{-\infty}^{+\infty} f(t) \cdot \phi_{j, k}(t) \cdot d t=<f(t), \phi_{j, k}(t)>
$$

Since these spaces are nested and complete for all finite energy signals, then as $j \rightarrow \infty$ the error between $f(t)$ and its approximation $f_{j}(t)$ approaches zero. That is, the space $\mathrm{V}_{\mathrm{j}+1}$ can better approximate $f(t)$ than $\mathrm{V}_{\mathrm{j}}$ since it is more complete.

In order to have an efficient decomposition of a function into multiple signal components to be analyzed separately, a set of orthogonal and complete basis is needed. Therefore the analysis starts with this nested space representation and the definition of an orthogonal and complete set of subspaces in the following way. Let $\mathrm{W}_{\mathrm{j}}$ denote the complement of $\mathrm{V}_{\mathrm{j}+1}$ in $\mathrm{V}_{\mathrm{j}}$, where complementary means that

$$
\mathrm{V}_{\mathrm{j}}=\mathrm{V}_{\mathrm{j}-1}+\mathrm{W}_{\mathrm{j}-1} \text { and } \mathrm{V}_{\mathrm{j}-1} \cap \mathrm{W}_{\mathrm{j}-1}=\{0\}
$$

Loosely speaking, this means that a subspace $\mathrm{W}_{\mathrm{j}-1}$ is exactly what is missing from the set $\mathrm{V}_{\mathrm{j}-1}$ to become $\mathrm{V}_{\mathrm{j}}$ and is therefore orthogonal to $\mathrm{V}_{\mathrm{j}-1}$. In this case, $\mathrm{V}_{-\mathrm{n}}, \mathrm{W}_{-\mathrm{n}}, \mathrm{W}_{-\mathrm{n}+1}, \ldots, \mathrm{W}_{0}, \mathrm{~W}_{1}, \ldots, \mathrm{W}_{\mathrm{j}}, \ldots, \mathrm{W}_{\mathrm{m}-1}$ form an orthogonal set and as complete as $\mathrm{V}_{\mathrm{m}}$. And since $\mathrm{V}_{\mathrm{m}}$ becomes more and more complete as $\mathrm{m}$ grows indefinitely so does the orthogonal basis just defined. In practice, in the discrete domain the level of approximation $\mathrm{m}$ of a signal is limited by the 
available sampling rate. The equivalent of this statement in Fourier theory is that we cannot recover information about the continuous signal for frequencies higher than the corresponding Nyquist frequency.

It can be shown that when $\phi(t)$ exists then each subspace $\mathrm{W}_{\mathrm{j}}$ has a basis function $\psi_{j, k}(t)=2^{\mathrm{j} / 2} \psi\left(2^{j} t-k\right)$ where the function $\psi(\mathrm{t})$ is called the mother wavelet and satisfies the following equation called the wavelet equation

$$
\psi(t)=2 \cdot \sum_{k=0}^{N} h_{1}[k] . \phi(2 t-k)
$$

where

$$
\sum_{k=1}^{N}\left|h_{1}(k)\right|=1
$$

so that $\psi(t)$ is normalized to 1 . It can also be shown from the wavelet and refinement equations that the mother wavelet can also be synthesized at any scale using the FIR filters with coefficients $h_{0}(k)$ and $h_{l}(k)$. The conditions for the existence of $\phi(t)$ and $\psi(t)$ are discussed in References $1,2,3$, and are beyond the scope of this paper. For now it is assumed that these functions do exist for some set of coefficients $h_{0}(k)$ and $h_{l}(k)$ according to the above formulation and that they satisfy equations (2) and (6).

For $\mathrm{n}=0$ the orthonormal set becomes:

$$
\mathrm{B}=\left\{\mathrm{v}_{0}, \mathrm{w}_{0}, \mathrm{w}_{1}, \ldots, \mathrm{w}_{\mathrm{j}}, \ldots, \mathrm{w}_{\mathrm{m}-1}\right\}
$$

Therefore any function $f(t)$ in $L^{2}(R)$ can be approximated in $\mathrm{V}_{\mathrm{m}}$ as follows

$$
f_{m}(t)=\sum_{k=0}^{N} a_{0}(k) \cdot \phi(t-k)+\sum_{j=0}^{m-1} \sum_{k=0}^{N} b_{j}(k) \cdot \psi_{j, k}(t)
$$

where

$$
a_{0}(k)=<f_{m}(t), \phi_{k}(t)>\text { and } b_{j}(k)=<f_{m}(t), \psi_{j, k}(t)>
$$

Equation (8) represents the projections of the function $f(t)$ into orthonormal wavelet spaces and an approximation at $\mathrm{V}_{0}$. The first term in equation (8) is the low resolution approximation of $f(t)$ in $\mathrm{V}_{0}$. For the second term of the same equation, as $\mathrm{j}$ increases, the basis functions $\psi_{j, k}(t)$ become more and more compressed and localized in time and therefore called the wavelet details. As $m$ increases $f_{m}(t)$ converges to $f(t)$. In practice, in the discrete domain, the largest achievable $\mathrm{m}$ is limited by the sampling rate. This is almost intuitive, because in general when a function is sampled, unrecoverable details were lost. However if the sampling rate is high enough the approximation becomes exact for all practical purposes. This is similar to the relationship between the Nyquist frequency and the sampling rate in Fourier analysis.

In signal processing all signals are discrete, and therefore the equations above cannot be directly applied. In the rest of this section the relationships between the coefficients $h_{0}(k), h_{l}(k)$ and the functions $\phi(t)$ and $\psi(t)$ are examined. The properties and constraints on these coefficients are introduced, followed by the presentation on how the wavelet decomposition of a function according to (8) can be approximated by calculating the coefficients in (9) using a filter bank with the low pass filter $\mathrm{h}_{0}(\mathrm{k})$ and the high pass filter $h_{l}(k)$.

First, it can be shown that the following orthogonality conditions on the wavelets and scaling functions are equivalent to orthogonality conditions on the FIR filters $h_{0}(k)$ and $h_{l}(k)$ outlined in equation set (11). 


$$
\begin{aligned}
& <\phi_{k}(t), \phi_{l}(t)>=\delta(k-l) \\
& <\phi_{k}(t), \psi_{j, l}(t)>=0 \\
& <\psi_{i, k}(t), \psi_{j, l}(t)>=\delta(i-j) \delta(k-l)
\end{aligned}
$$

The corresponding orthogonality conditions on the filter coefficients are

$$
\begin{aligned}
& \sum_{n=1}^{N} h_{0}(n) \cdot h_{0}(n-2 k)=\delta[k] \\
& \sum_{n=1}^{N} h_{0}(n) \cdot h_{1}(n-2 k)=0 \\
& \sum_{n=1}^{N} h_{1}(n) \cdot h_{1}(n-2 k)=\delta[k]
\end{aligned}
$$

If $h_{0}(n)$ is known to satisfy the first of these equations, and if $h_{1}(k)$ is chosen to be defined according to Eq. (12) then the rest of the equations in Eq. (11) are satisfied. Therefore, knowing $h_{0}(k), h_{l}(k)$ can be computed with ease as the two sets of coefficients define orthogonal filters according to Eq. (11).

$$
h_{1}(n)=(-1)^{n} \cdot h_{0}(N-n)
$$

Furthermore, it can also be shown using the refinement and wavelet equations that the wavelet coefficients defined in Eq. (9) can be calculated using these filters according to the following equations called multiresolution decomposition equations

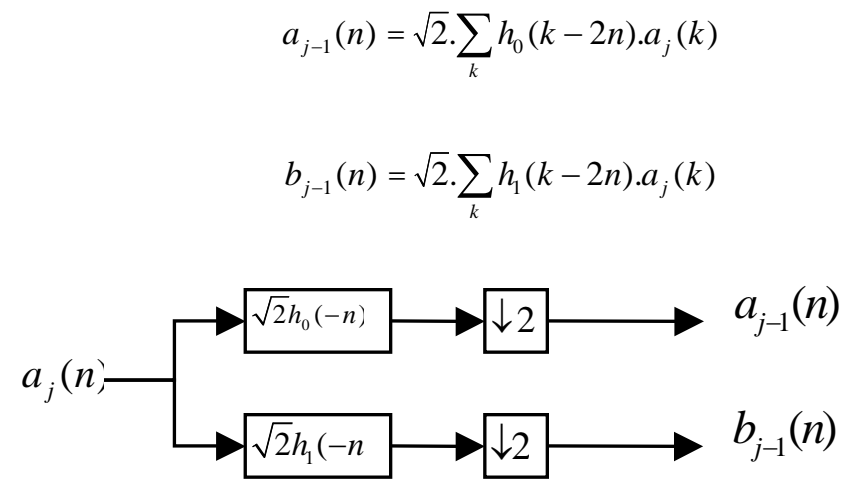

Figure 1. Computation of wavelet coefficients using an analysis bank

So, given signal samples $f_{s}(n)=f\left(n . T_{s}\right)$ of the function $f(t)$, these samples could be thought of as samples of $f_{m}(t)$ $=f(t)$ smoothed with a kernel $\phi_{m}(t)$, the basis function of $\mathrm{V}_{\mathrm{m}}$, as shown in Eq. (15). In practice, this is not a bad assumption since the output of any A/D process involves smoothing of the analog signal by a narrow time window before digitization. When the sampling period is normalized to 1 , equation (15) is nothing but the coefficients $\mathrm{a}_{\mathrm{m}}(\mathrm{k})$. So starting with the sampled signal, Eq. (13) and Eq. (14) can be used recursively to find the coefficients of the wavelet decomposition of the signal $f(t)$.

$$
f_{m}\left(n . T_{s}\right)=<f(t), \phi_{m}(t-n . T s)>
$$

It can also be shown that the signal can be reconstructed from the wavelet coefficients using the synthesis filter bank shown in Fig. 2 which is the implementation of Eq. (16). This equation is the multiresolution reconstruction equation. Given the orthogonality conditions, this equation is obtained after imposing one more condition called the perfect reconstruction condition on the analysis and synthesis filters ${ }^{1,2,3}$. 
$a_{j}(n)=\sqrt{2} \cdot \sum_{k} a_{j-1}(k) \cdot h_{0}(n-2 k)+\sqrt{2} \cdot \sum_{k} b_{j-1}(k) \cdot h_{1}(n-2 k)$

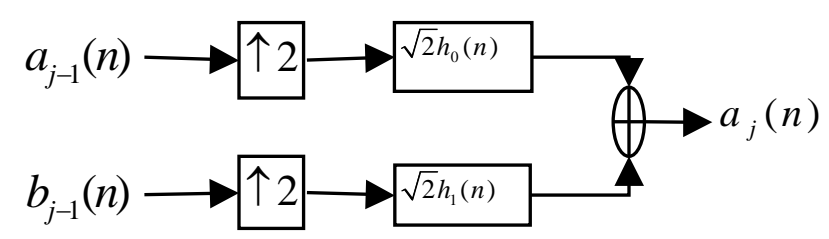

Figure 2. Signal reconstruction using complementary synthesis filter bank

Because of the restrictions imposed by the orthogonality conditions on the analysis filter bank and by the perfect reconstruction conditions on both the analysis and synthesis filters, it can be seen by substituting Eq. (12) in Eq. (14) and Eq. (16) that all four filters are completely defined once $h_{0}(n)$ is known. Therefore, the scaling function, and wavelets and the wavelet decomposition are all defined once the coefficients $h_{0}(n)$ are known. The process of finding this filter's coefficients is the subject of wavelet design and is beyond the scope of this paper. By redefining the nomenclatures for these filters to simplify notations, the analysis filter bank is reintroduced as,

$$
\begin{aligned}
& H_{0}(n)=\sqrt{2} \cdot h_{0}(-n) \\
& H_{1}(n)=\sqrt{2} \cdot h_{1}(-n)
\end{aligned}
$$

and the synthesis filter bank is redefined as,

$$
\begin{aligned}
& F_{0}(n)=\sqrt{2} \cdot h_{0}(n) \\
& F_{1}(n)=\sqrt{2} \cdot h_{1}(n)
\end{aligned}
$$

In summary, the wavelet decomposition of a continuous signal can be approximated by recursive filtering and decimation of the sampled signal through the filter bank in Fig. 1. Conversely, the sampled signal can be synthesized from the wavelet coefficients by recursively applying the synthesis bank on the wavelet and approximation coefficients $a_{0}(k), b_{0}(k), b_{1}(k), b_{2}(k) \ldots b_{m-l}(k)$. The full decomposition and reconstruction process is illustrated in Fig. 3 for $m=2$.

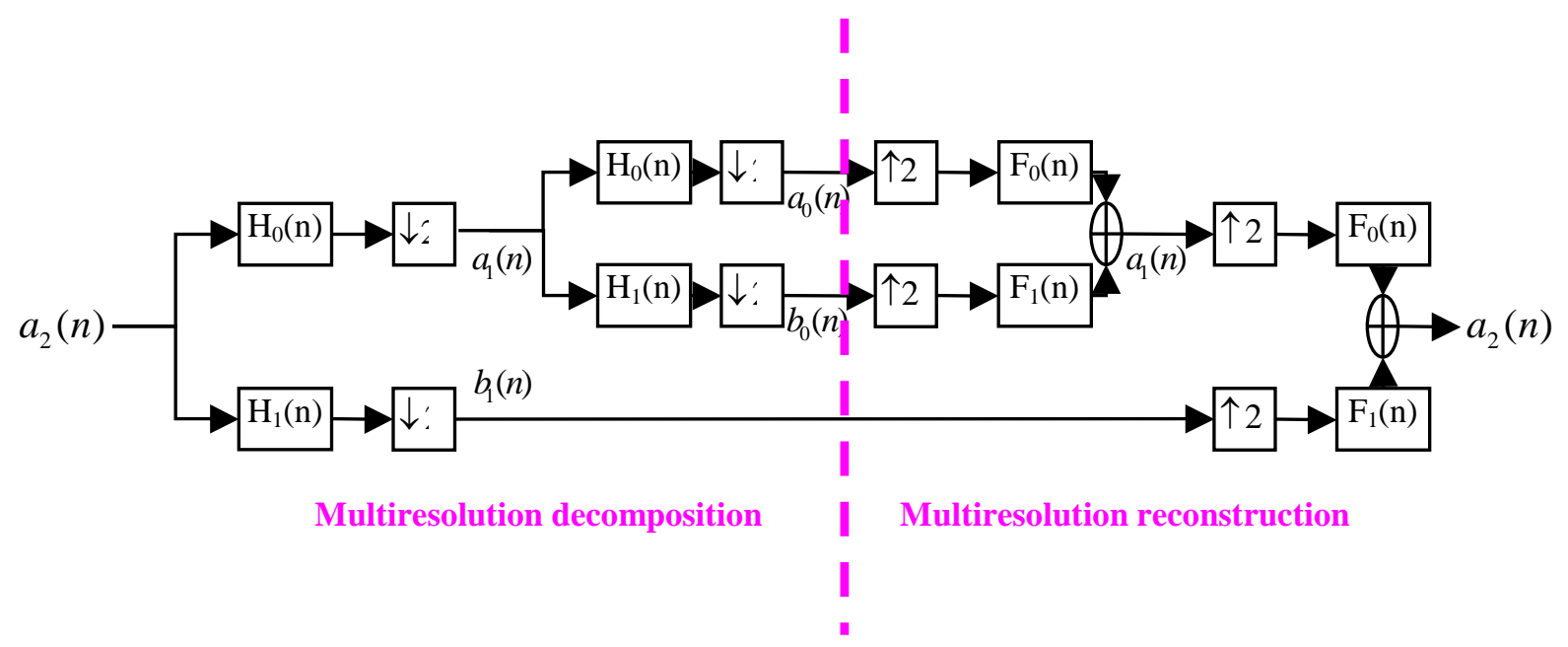

Figure 3. Full decomposition and reconstruction wavelet tree for $\mathbf{m}=2$ 
By removing the summation nodes from the tree, the low resolution approximation of the signal and its projections on the wavelet subspaces can be reconstructed so they can be processed independently. This is shown in Fig. 4 for $\mathrm{m}=2$ for illustration purposes but could be done for any size orthonormal basis. As will be shown later, the collection of synthesized signal projections from all microphones on a particular wavelet space will be processed through a time domain beamformer to produce an image of the wake corresponding to that wavelet space.

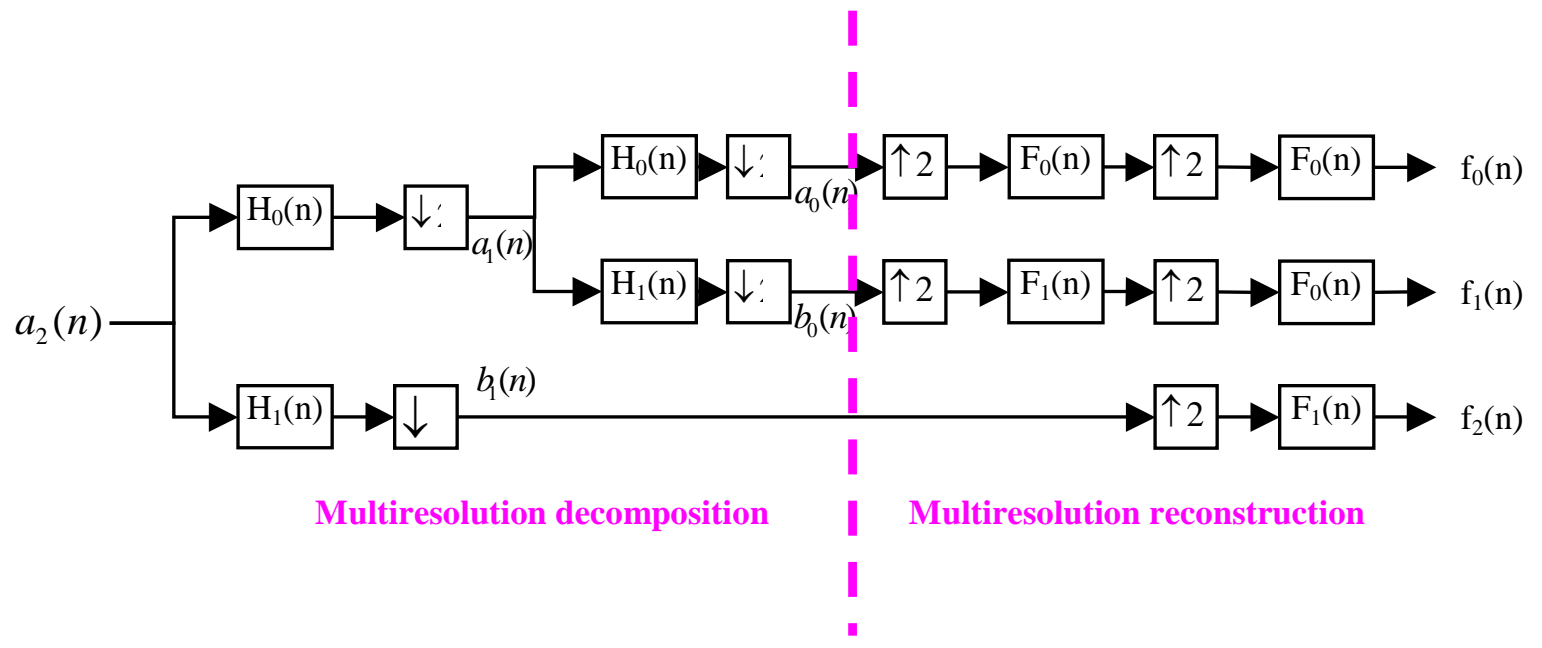

Figure 4. Multiresolution decomposition of the sampled signal, and reconstruction of the low resolution signal approximation $f_{0}(n)$ as well as the individual signal projections on each of the wavelet subspaces for $m=2$

In the next section, the discussion of time-domain beamforming and the illustration on how it can be integrated with the wavelet analysis for the purpose of imaging the wake in each of the wavelet subspaces are presented.

\section{B. Beamforming in Wavelet Subspaces}

Let $s(\vec{x}, t)$ be a sound source distribution and $p\left(\vec{x}_{i}, t\right)$ be the pressure sensed by the microphone at location $\vec{x}_{i}$. Assuming that each infinitesimal portion of the source distribution $s(\vec{x}, t) . d v$ is a simple monopole emitting spherical waves in a homogeneous atmosphere with negligible attenuation, we get:

$$
p(\vec{x} i, t)=\int \frac{1}{R_{i}(\vec{x})} s\left(\vec{x}, t-\frac{R_{i}(\vec{x})}{c}\right) \cdot d \vec{x}
$$

where

$$
R_{i}(\vec{x})=|\vec{x}-\vec{x} i|
$$

When the primary focus is in mapping the acoustic power distribution of a finite square horizontal plane at some altitude $\mathrm{Z}_{0}$ above the array during a time interval $T$, this mapping can be carried out as follows. First, consider a grid on that plane and estimate the average acoustic power emitted by each grid point over the same time interval $T$. An image is then constructed with each pixel-intensity being a function of the average power emitted from the corresponding grid point in the source plane.

The average power corresponding to a particular location $\vec{x}_{0}$ on the grid is estimated by focusing the microphone array on that grid point and averaging the power of the resulting signal over the time interval $\mathrm{T}$. The array is focused 
by compensating for time delays in each of the microphone signals so that the signal corresponding to the sound emitted from the desired location is amplified. More precisely, a delay term, $\Delta t_{i}\left(\vec{x}_{0}\right)$ is defined as follows,

$$
\Delta t_{i}\left(\vec{x}_{0}\right)=-\frac{\left|\vec{x}_{i}-\vec{x}_{0}\right|}{c}
$$

After introducing this delay to each of the microphone signals and forming the sum the resulting signal is proportional to

$$
\hat{s}_{0}(t)=\frac{1}{M} \sum_{i=1}^{M} p\left(\vec{x}_{i}, t-\Delta t_{i}\left(\vec{x}_{0}\right)\right)
$$

Substituting Eq. (19) in (22) and interchanging the order of integration and summation, it follows that

$$
\hat{S}_{0}(t)=\frac{1}{M} \int\left\{\frac{1}{R_{i}(\vec{x})} \cdot \sum_{i=1}^{M} s\left(\vec{x}, t-\frac{R_{i}(\vec{x})}{c}-\Delta t_{i}\left(\vec{x}_{0}\right)\right)\right\} d \vec{x}
$$

By examining this equation, it can be observed that when $\vec{x}$ approaches $\vec{x}_{0}$ the sum inside the curly braces reduces to $M . s\left(\vec{x}_{0}, t\right)$. As the point of interest moves away from the location $\vec{x}_{0}$ the time delay term inside the parenthesis becomes more and more dependent on the microphone location and the signal may no longer add constructively. Therefore, this way of summing the microphone signals amplifies the acoustic signal corresponding to location $\vec{x}_{0}$ more than any other location. The estimated average power corresponding to the grid point $\vec{x}_{0}$ is therefore

$$
I_{0}=\int_{0}^{T}\left[\hat{s}_{0}(t)\right]^{2} \cdot d t
$$

How fast the response drops as one moves away from $\vec{x}_{0}$ depends on the array design and the level of correlation between the monopoles in the source distribution. This is the subject of array design which is beyond the scope of this paper. In general, the selectivity of the delay and sum beamforming improves as the array size and the number of microphones increases, and as sources become less correlated. Array designs are usually optimized by simulating the array response for various configurations. If the process of obtaining equation (24) is repeated for each grid point, these results can be combined into an image representing the Acoustic Intensity Distribution Map (AIDM) of the sources intersecting the horizontal plane being examined.

Just as this beamforming process is performed by processing the broadband signals, it could be equally applied to orthogonal projection of that signal on the wavelet subspaces. That is, one can beamform the projections of all microphone signals on the same subspace to generate an AIDM in that subspace. So for a time interval $T$, $\mathrm{m}$ images are formed, where $m$ is the number of subspaces in (7). This full process is depicted in Fig. (5). Since these images represent the average power of signals from an orthogonal and complete set, they can be added to reconstruct the original broadband image or reconstruct an image selected sub-bands without introducing distortions. This is because the power of the sum of two orthogonal functions is the sum of the powers. That is, if $f(t)$ and $g(t)$ are 2 orthogonal functions and if the time averaging were denoted by <.> then the power of the sum is

$$
\left\langle(f(t)+g(t))^{2}\right\rangle=\left\langle f(t)^{2}\right\rangle+\left\langle g(t)^{2}\right\rangle+2\langle f(t), g(t)\rangle=\left\langle f(t)^{2}\right\rangle+\left\langle g(t)^{2}\right\rangle
$$


Therefore the value of the average power of a grid point in one of the sub-spaces can be added to the average power of the same grid point in another subspace to get the average power of the that grid point as if beamforming was done on a signal synthesized from both subspaces. This eliminates the need to re-beamform to reconstruct images from any combination of sub-spaces or sub-bands while guaranteeing no signal distortion.

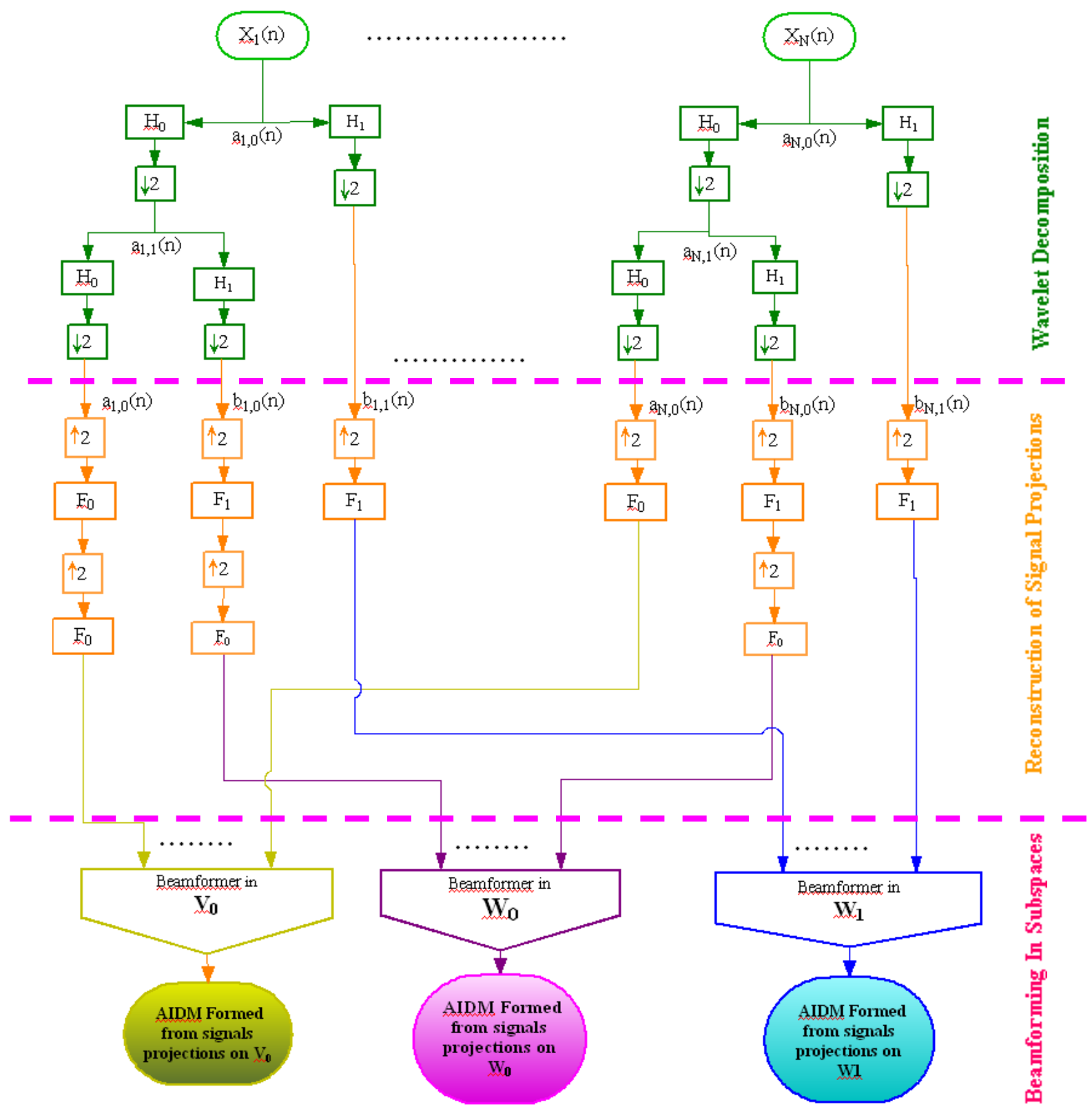

Figure 5. Flow chart illustrating the process of performing $m=2$ wavelet decomposition of $\mathbf{N}$ microphone signals, projecting each of the signals in the approximation space $\mathrm{V}_{0}$ and the wavelet spaces $W_{0}$ and $W_{1}$, then finally beamforming the projections of all signals on the same subspace to generate an AIDM corresponding to this particular projection. Since for this illustration we have $m=2$, therefore we get 3 AIDMs for each processing time interval. 


\section{Application and Results}

\section{Sample Application}

In this section, the applicability of the developed technique to reveal wake vortices' signal composition is illustrated. The image recomposition from selected subspaces is then shown next, where the selection process is based on particular criteria. This is followed by an approach on how this selection process might be automated using image processing algorithms. In the following example, data from the 252-element NASA-Volpe microphone array deployed in Denver during September of 2003 is considered ${ }^{1,2,5,6,9}$. This particular case refers to a landing 757 aircraft. Fig. 6 is the AIDM generated from beamforming on a horizontal grid at $500 \mathrm{ft}$ altitude and with a size of $1500 \mathrm{ft}(\mathrm{E}-\mathrm{W})$ and $1000 \mathrm{ft}(\mathrm{N}-\mathrm{S})$. The time is about 8 seconds after the aircraft passed above the array center and the integration time for the acoustic power is 2 seconds. The microphone signals are first filtered down to $400 \mathrm{~Hz}$ using 8-th order Chebychev low-pass filter and an image representing the AIDM in a 2 -second time interval is generated using conventional time-domain beamforming as discussed in section II-B. This image is shown in Fig. 6

As discussed previously, this image represents the integrated acoustic power in the entire $400 \mathrm{~Hz}$ band and provides no information on the wake acoustic spectral content in that time interval. Should further detail on the spectral content be desired in the AIDM, it is necessary to either filter the time series data using band-pass filters in the case of time-domain beamforming, or beamform in very narrow bands in the case of frequency domain beamforming. For the first case, if the filters designed are not orthogonal, redundant information will exist in adjacent bands due to spectral leakage. If the filters are sharp enough to be orthogonal but they do not form a complete basis for the analysis band, then there will be missing information or distortions in the transition bands when reconstruct of the signal from successive bands is attempted. These problems are solved by the orthogonality and PR properties of wavelet decomposition. In the case of frequency domain beamforming, a large number of beamforming steps are needed to cover the whole signal-band since it is constrained by a predefined high spectral resolution due to the dependence of the steering vector on frequency. The proposed wavelet based approach uses the flexibility of multiresolution analysis to decompose the signal into subspaces or pseudo-spectral components. The term pseudo-bands or pseudo-spectrum is used because the wavelet decomposition is not equivalent to band-pass filtering. Although every projection is practically band-limited, the spectrum in that band looks like an aliased or distorted version of a clean bandpassed version of that signal. They look that way because in this contest they are being observed with a Fourier view. However these distorted band-passed like

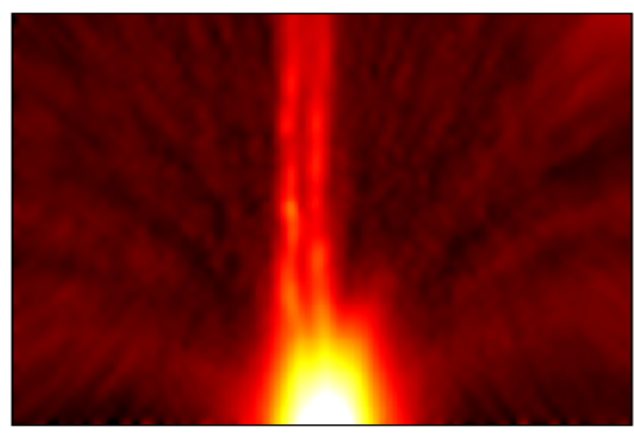

Figure 6. AIDM for the wake of a 757 airplane using conventional time-domain beamforming in the time interval $[7,9]$ seconds relative to airplane overhead

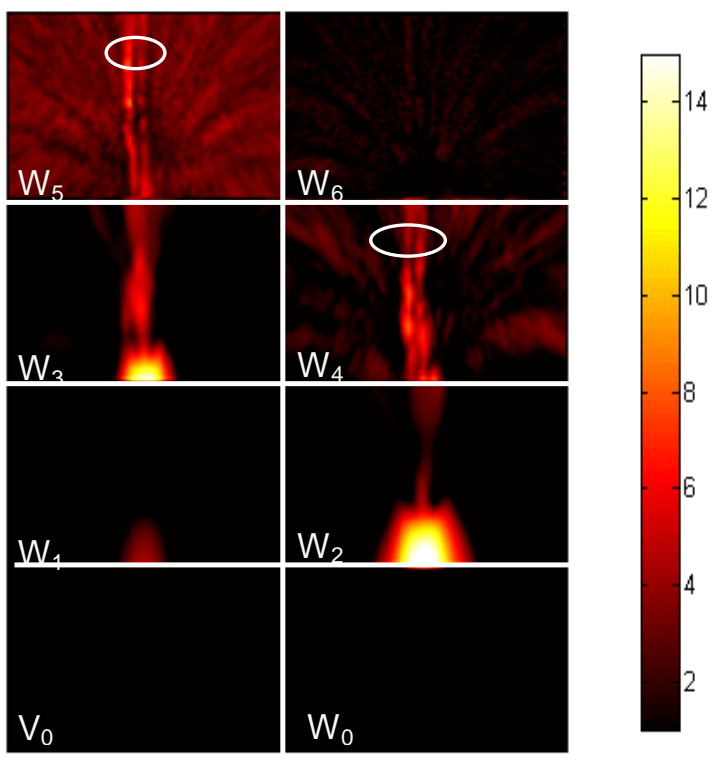

Figure 7. AIDM from beamforming using individual projections of the microphone signals onto wavelet subspaces. The time interval is the same $[7,9]$ seconds relative to airplane overhead time used in the previous figure signals are orthogonal projections which contain all the information about the signal and can be used to reconstruct it. 
Fig. 7 shows the result of beamforming after projecting each of the microphone signals on multiple wavelet subspaces. The filter $\mathrm{h}_{0}(\mathrm{k})$ in this wavelet decomposition was the 10-th order Symmlet. The number of wavelet levels in the decomposition is $m=7$. Beamforming was performed in the approximation space $\mathrm{V}_{0}$, and in each of the wavelet subspaces separately to generate $m+1=8$ images. The one labeled $\mathrm{V}_{0}$ represents the AIDM produced using the projection of the microphone signals on the approximation space, and $\mathrm{W}_{1}$ is that of the projections on the coarsest wavelet subspace. The images labeled $\mathrm{W}_{\mathrm{i}}$ are results from beamforming in the $\mathrm{i}$-th wavelet space. As can be observed from these images, the acoustic energy of the wake in the $T=2$ seconds time-interval is concentrated in the wavelet subspaces $\mathrm{W}_{3}, \mathrm{~W}_{4}$, and $\mathrm{W}_{5}$. It could also be seen that the power level of the acoustic waves emitted by the wake is not the same in these 3 subspaces. It is also not consistently higher in one scale (or subspace) relative to the other. For example, in Fig. 7, considering the beamformed projection on $\mathrm{W}_{4}$ of the microphone signals, the region of the right wake enclosed in the white circle is brighter than its counterpart in the image produced by the beamformed projection of the signals on $\mathrm{W}_{5}$. However, the opposite is true for the left wake; that is, the region of the right wake enclosed in $\mathrm{W}_{5}$ is brighter. Also different features of the wakes are apparent in each of the spaces. This could in part be due to the way the wake emits sound in a particular wavelet space, and in another part due to the shape of the array point spread function in that wavelet space.

Now, assume that it is desired to form an image only from subspaces where the airplane does not appear and where the integrated power in the wake region has a strong enough SNR relative to the airplane and the rest of the noise (i.e. above a certain SNR-dependent threshold for each frame). Of course depending on the application, other criteria could be selected (such as the appearance of 2 wakes as opposed to one wake in the frame), but this criterion was selected for the purpose of illustration and because it is relatively simple to devise an image processing algorithm to automate it. Looking at the frames of Fig. 7 it is obvious that the frames that meet these criteria are the ones corresponding to $\mathrm{W}_{5}$ and $\mathrm{W}_{6}$. Therefore the resulting image should be recomposed by re-beamforming the microphone signals projected on these two subspaces. However, since these images are the logarithm of the integrated signal powers, and since all subspaces are orthogonal, by using equation (25) it can be observed that this recomposition can be done in the following 3 simple steps:

- First, use the inverse logarithms of the 2 Frames corresponding to the desired subspaces $\mathrm{W}_{5}$ and $\mathrm{W}_{6}$.

- $\quad$ Add the two resulting frames.

- Take the logarithm of the resulting sum.

The resulting image along with the original broadband image is shown in Fig. 8. In general, as discussed earlier in this section, if signals were decomposed using Fourier-based band-pass filters that do not satisfy the orthogonality and PR conditions offered by wavelets. this process of decomposition would not be valid.

The image processing steps used to find the frames containing the wake and segment it out from the region of the image corresponding to the airplane and other noise artifacts (which are the information needed to make the decision on which frames to use for image recomposition) are shown in Fig. 9. This image processing is an

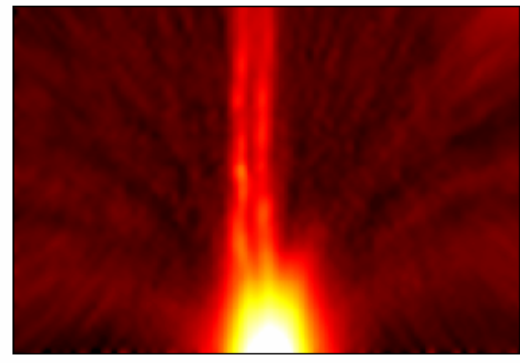

8-(a)
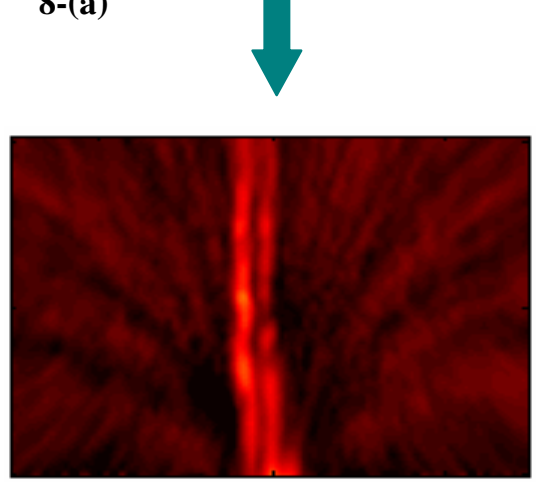

8-(b)

Figure 8. (a) Original image from beamforming in the full band. (b) Image recomposed from signal projections on subspaces $W_{5}$ and $\mathbf{W}_{6}$. example of how to automate the decision on which frames to use for image reconstruction, and as bi-product it can segment out the image into one where the wake alone is present and another containing the rest of the noise as shown in Fig. 9-(e) and 9-(f).

The frames of the decomposed image of Fig. 9-(a) are first thresholded to generate a mask corresponding to the wake and airplane regions when they exist as seen in Fig. 9-(b). This figure shows that this process picks up some 
noise or acoustic power other than that corresponding to the wake and airplane location. In the next step, an image processing algorithm called Component Labeling ${ }^{10}$ is applied, which identifies how many continuous objects there are in an image and label each of them. For each component the algorithm calculates its width and removes the portions where the width exceeds some preset threshold. This algorithm assumes that the airplane will manifest itself as a wide object that might or might not appear to be connected to the wake. Therefore, places where the width is larger than the threshold most probably belong to the airplane region and are removed from the mask. The result of this process is the generation of a mask containing the airplane regions. A mask containing only the wake and all the other noise except that corresponding to the airplane region can now be generated, and is shown in 9-(c). Finally to remove the rest of the noise, the image is convolved with a narrow vertical rectangular structure element and thresholded, the result is shown in Fig. 9-(d). This figure shows the masks corresponding only to the wake regions in the frames where the wake has significant acoustic energy. The result of using these masks to segment out the wake from the rest of the image is shown in Fig. 9-(e) and Fig. 9-(f).

Now that the algorithm knows that the only frames that contain the wake but not the airplanes are $\mathrm{W}_{5}$ and $\mathrm{W}_{6}$, and because of the orthogonality property they can be added to generate Fig. 8-(b) as mentioned earlier in this section. 


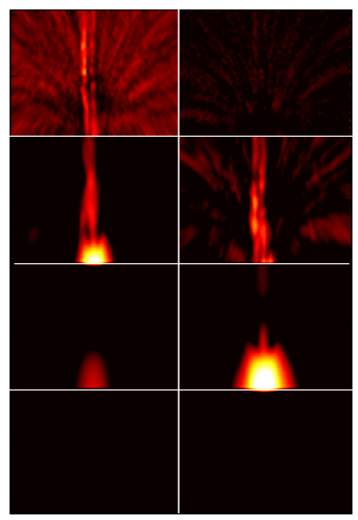

9-(a)

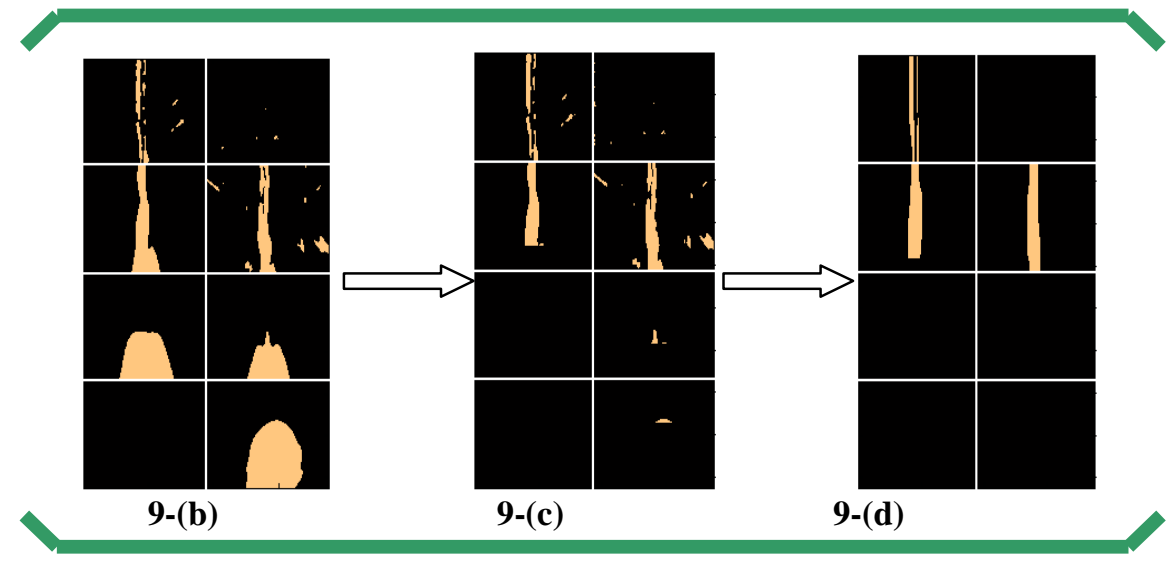

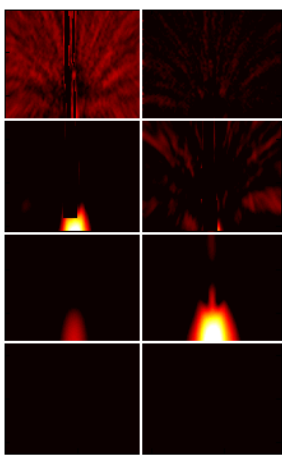

9-(e)
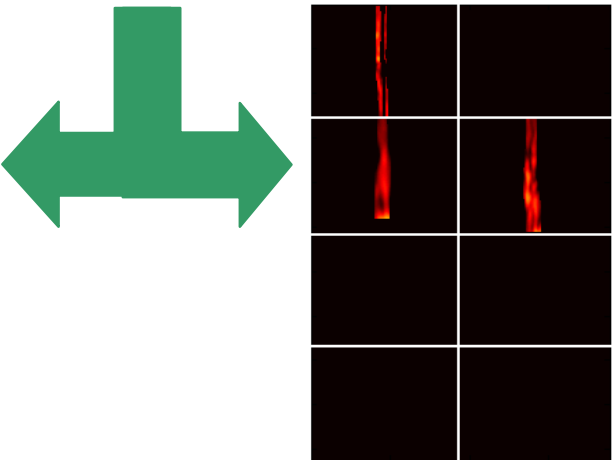

9-(f)

Figure 9. (a) Image formed from beamforming in wavelet sub-spaces. (b) Thresholded image based on the SNR for each frame. (c)Masks created after detecting and removing the airplane regions using component labeling and horizontal projection of each component. (d) Masks representing the wake regions only. (e) Resulting image projections containing everything except the wake region. (f) Resulting image projections with the wake regions only. 


\section{Validation Against Fourier Based Filters}

In this section, an approximation to the multi-resolution decomposition using 9 band-pass FIR filters with nonuniform resolution is generated and shown in Fig. 10. As discussed earlier in this paper, the narrower the band the longer the filter length is and the slower the processing becomes. So each microphone signal was first filtered with these 8 band-pass filters, followed by beamforming in each sub-band to generate approximations to the images from the wavelet decomposition. The center of each band-pass filter and the cutoff frequencies approximate the corresponding wavelet basis approximate bandwidth and center frequency (sometimes referred to as pseudofrequency).

As can be observed in Fig. 11, the results from approximating the wavelet decomposition with band-pass filters compare very well to the wavelet decomposition with a shorter orthogonal filter bank. An important note is that the analog signals were filtered using a highpass filter with a lower cutoff frequency of $20 \mathrm{~Hz}$. Therefore the comparison of wake signal power in the last 4 sub-bands (and the last 4 subspaces or pseudo-bands) to the upper 4 is not a fair comparison but it was done to illustrate the technique. Finally, we observe that the similarity between the 2 decompositions is striking as if the wavelet view is no different from the Fourier-based approach. It is believed that this is due to the fact that the signal was decimated to 1024 samples per seconds after filtering it down to $400 \mathrm{~Hz}$ prior to the wavelet decomposition. If we had started with the full signal band that has a sampling rate of $25.6 \mathrm{KHz}$, we believe that dissimilarities would have been more pronounced. In fact these images are not exactly the same and to illustrate that images delineating the difference were made by subtracting Fig. 11-(b) from Fig. 11-(a). The result is shown in Fig. 12. It can be observed that the lower images are different from the upper ones. This supports the previous statement about the image becoming more different for higher order decomposition. This will be the subject of future investigation. For now, this analysis is sufficient for the purpose of validating the present wavelet based

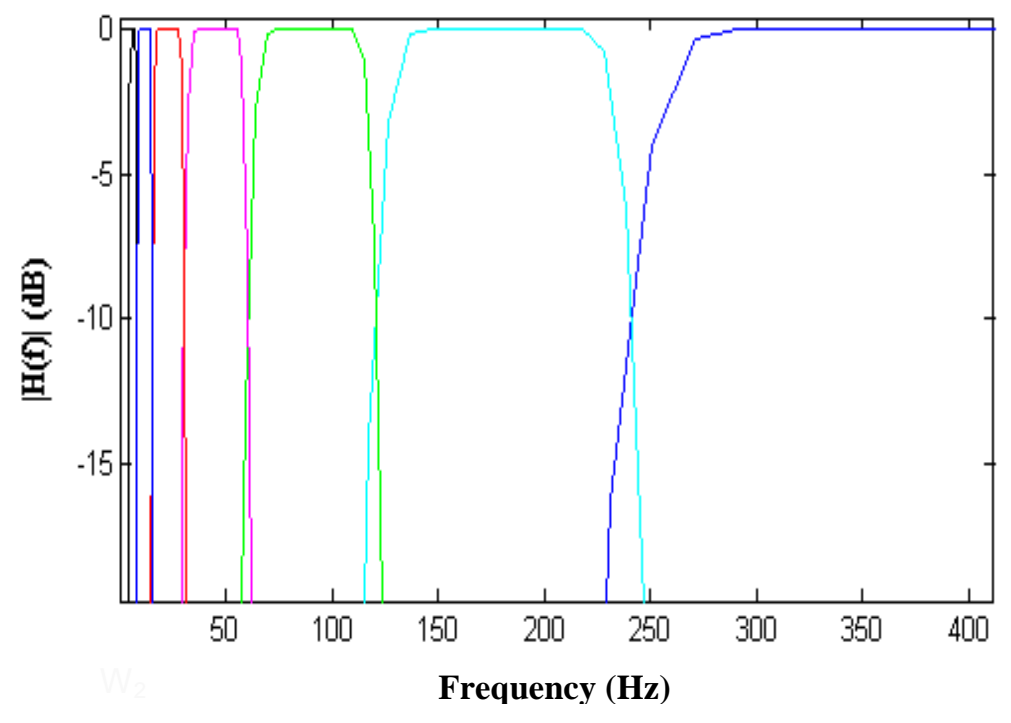

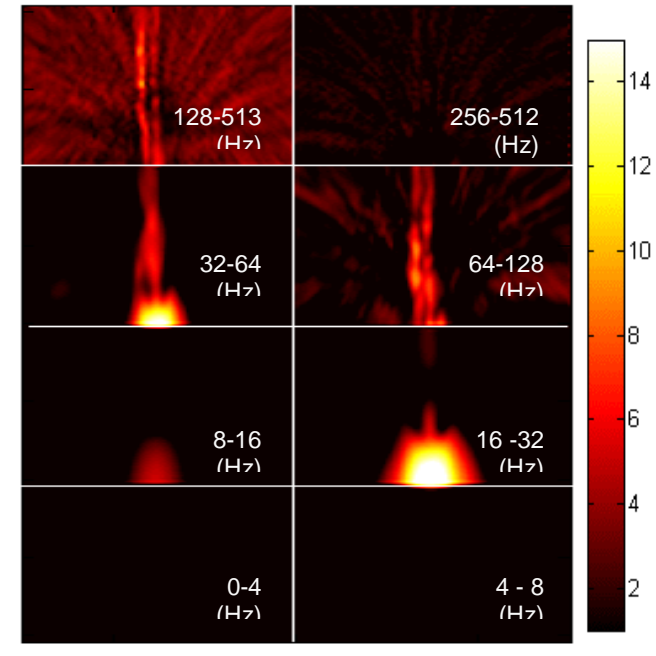

(a)

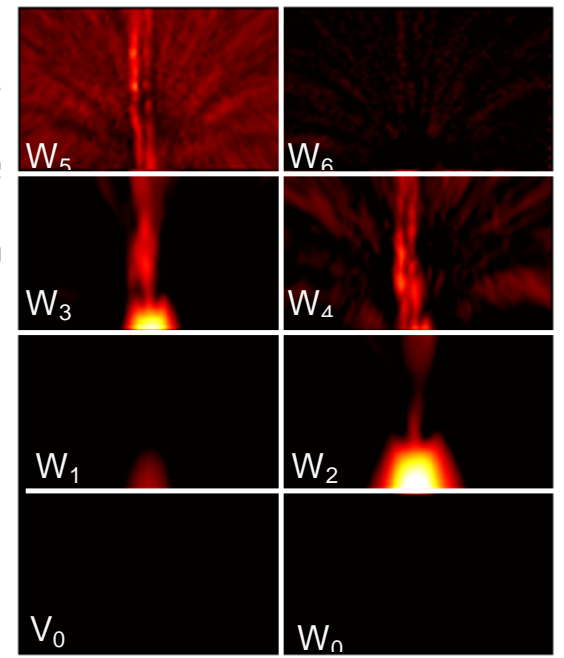

(b)

Figure 11. Validation of the wavelet multi-resolution analysis. (a) Frames from beamforming in multiresolution sub-bands using long band pass filters with the frequency responses depicted in Fig. 11. (b) Frames from beamforming in wavelet subspaces. 
technique against the Fourier based one.

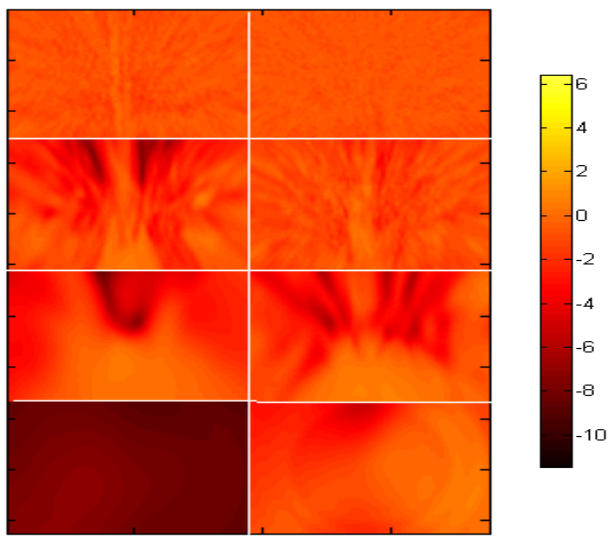

Figure 12. Difference image Fig. 11-(b) minus Fig. 11-(a). The frames corresponding to lower subspaces show larger differences. That is, the deeper the decomposition the more the wavelet representation becomes different than the decomposition with Fourier-based filters

\section{Conclusion}

The present paper presents an approach that combines wavelet analysis with beamforming to provide visual and quantitative assessment of the wake's signal composition corresponding to a processing time interval $T$. While previous approaches use time domain beamforming to look at the broadband signal or at selected very narrow bands (as is the case of frequency domain beamforming), this technique uses the multiresolution, orthogonality and perfect reconstruction capabilities of the wavelet decomposition to analyze the full signal composition in orthogonal subspaces. The use of this technique to perform image reconstruction from combining selected subspaces was also discussed. The illustration of how image processing algorithms can be used to automatically select the subspaces from which to perform image reconstruction has also been demonstrated. The advantages of using this technique over Fourier-based techniques as well as a validation against a Fourier based approach were also discussed. However, since the wavelet decomposition was done after low-pass filtering the microphone signals, the assessment of this approach is not complete. In the future, reprocess the data without low-pass filtering and redo the comparison is planned. Furthermore, future plans include assessing the impact of using a different basis function as opposed to the one used in this paper, as well as adding more flexibility to the multi-resolution analysis by using wavelet packets that offer greater flexibility when higher resolution information is needed about a particular band while maintaining orthogonality of the signal components for efficient analysis. Other possible extensions of this work are the iteration between image processing and wavelet-packet decomposition for adaptive decision making on which sub-band should be further decomposed. It is anticipated that as the consequence, refined spatial and temporal resolution of the AIDM as well as the enhanced ability to visualize vortex dynamics would lead to advancement in better understanding of aircraft wake vortices at various stages of development and interaction with the environment.

\section{Acknowledgements}

The authors wish to express their appreciation to the NASA's Aeronautics Research Mission Directorate, and in particular the Airspace Systems Program, for funding the exploration of this fundamental mechanism for detecting aircraft wakes, and to the program manager, Wayne Bryant, for ensuring that the work focused on addressing the core issues. 


\section{References}

${ }^{1}$ Strang, G. and Nguyen, T., Wavelets and Filter Banks, Wellesley-Cambridge Press, 1996.

${ }^{2}$ Mallat, S., A Wavelet Tour of Signal Processing, Academic Press, 1999.

${ }^{3}$ Daubechies, I., Ten Lecture on Wavelets, Society for Industrial and Applied Mathematics, 2004.

${ }^{4}$ Donoho, D. L., "Denoising Via Soft Thresholding," IEEE Transactions on Information Theory 41, pp. 613-627, May 1995.

${ }^{5}$ Dougherty, R. P., Wang, F., Booth, E., Watts, M., Fenichel, N., and D’Errico, R., “Aircraft Wake Vortex Measurements at Denver Airport," AIAA Paper 2004-2880.

${ }^{6}$ Wang, F., Wassaf, H., Dougherty, R., Clark, K., Gulsrud, A., Fenichel, N., and Bryant, W., "Passive Wake Acoustics Measurements at Denver International Airport," 4th NASA ICNS Conference, Fairfax, VA, April 2004.

${ }^{7}$ U. Michel, and P. Böhning, "Investigation of Aircraft Wake Vortices with Phased Microphone Arrays," AIAA Paper 2002-2502, Breckenridge, CO, June 2002.

${ }^{8}$ Dougherty, R.P., "Extensions of DAMAS and Benefits and Limitations of Deconvolution in Beamforming," AIAA Paper 2005-2961, May 2005.

${ }^{9}$ Booth, E. R. Jr., and Humphreys, W. M. Jr., "Tracking and Characterization of Aircraft Wakes Using Acoustic and Lidar Measurements," AIAA Paper 2005-2964, Monterey, California, May 2005.

${ }^{10}$ Gonzalez, R.C., and Woods, R. E., Digital Image Processing, Prentice-Hall, Inc., New Jersey, 2002.

${ }^{11}$ Johnson, D. H., and Dudgeon, D. E., Array Signal Processing Concepts and Techniques, Prentice Hall, New Jersey, 1993. 